\title{
Hermes results on 3D imaging of the nucleon
}

\author{
Luciano L. Pappalardo \\ (on behalf of the Hermes Collaboration)
}

Dipartimento di Fisica e Scienze della Terra, Università degli Studi di Ferrara and INFN, Sezione di Ferrara, Via Saragat 1, 44122 Ferrara, Italy

\begin{abstract}
The study of the quantum phase-space distribution of quarks and gluons inside nucleons in terms of TMDs and GPDs has become, in the last decade, a cutting-edge research field in hadron physics. These non-perturbative objects, respectively measurable in semi-inclusive deep-inelastic scattering and exclusive processes, allow to obtain 3dimensional representations of the nucleon in the momentum and spatial coordinates as well as indirect insights into the still unknown parton orbital angular momentum. The HERMES experiment at HERA has been a precursor in this field. A selection of HERMES results sensitive to both TMDs and GPDs is presented.
\end{abstract}

Theoretical developments of the parton model in the framework of nonperturbative QCD followed by decades of deep-inelastic scattering (DIS) experiments have led to a relatively detailed description of the internal structure of nucleons in terms of quark and gluon degrees of freedom. In particular, very high-precision results on the longitudinal ${ }^{1}$ momentum distribution of quarks, described by the spin-independent parton distribution function (PDF) $f_{1}(x)$, are provided by global fits of world data [1] that span several orders of magnitude in $x$ and $Q^{2}$, the longitudinal momentum fraction of the nucleon carried by the partons and the squared four-momentum transfer of the hard probe to the struck quark, respectively. In the last decades an

\footnotetext{
${ }^{1}$ With respect to the direction of the exchanged virtual photon, the hard probe.
} 
increasing focus was put on the spin degrees of freedom of quarks and gluons, described by the spin-dependent helicity PDF $g_{1}(x)$. While a relatively precise knowledge of $g_{1}$ has been achieved for the valence quarks, large uncertainties still affect the results for sea quarks and gluons [1]. More recently, the HERMES experiment provided first evidences of a non-zero transverse spin distribution of up and down quarks, described by the transversity PDF $h_{1}(x)$, through the measurement of specific azimuthal asymmetries in the production of $\pi^{+}$and $\pi^{-}$(the so-called Collins effect) in semi-inclusive DIS (SIDIS) measurements off a transversely polarized hydrogen target [2]. The three fundamental PDFs $f_{1}(x), g_{1}(x)$ and $h_{1}(x)$ are said to be collinear, in the sense that they neglect any parton transverse momentum degree of free$\mathrm{dom}^{2}$, thus providing 1-dimensional descriptions of the nucleon structure in terms of the longitudinal momentum fraction $x$.

In recent years, huge efforts are being focused towards a more comprehensive multi-dimensional description of the nucleon. The final goal is to map the full quantum phase-space distribution of quarks and gluons inside the nucleon, as formally encoded by the Wigner function (depending on both spatial and momentum coordinates). Although, due to the uncertainty principle, the Wigner function is not directly measurable, two independent classes of partially integrated objects, the Transverse Momentum Dependent PDFs (TMDs) and the Generalized Parton Distributions (GPDs), are being intensively investigated both theoretically and experimentally. TMDs and GPDs allow for complementary descriptions of the nucleon in three dimensions (nucleon tomography), spanned by the quark's longitudinal momentum and, respectively, by its transverse momentum components and transverse spatial coordinates. Furthermore, they provide complementary insights into the yet unmeasured quark orbital angular momentum. Experimentally, TMDs and GPDs can be accessed through the analysis of specific azimuthal asymmetries measured, respectively, in SIDIS and hard exclusive processes, such as hard lepto-production of real photons or mesons.

At leading-twist, eight TMDs, each with a specific probabilistic interpretation in terms of quark number densities, enter the SIDIS cross-section in conjunction with a fragmentation function (FF) [3]: the poorly known chiral-odd Collins function $H_{1}^{\perp}$, describing left-right asymmetries in the fragmentation of transversely polarized quarks, or the relatively well known spin-independent chiral-even $D_{1} \mathrm{FF}$. Three of them are the transverse-

\footnotetext{
${ }^{2}$ More precisely, they are the only three leading-twist PDFs that survive integration over the parton momentum components that are transverse with respect to the direction of the hard probe.
} 
momentum-dependent versions of $f_{1}, g_{1}$ and $h_{1}$. The other five are the Sivers function $f_{1 T}^{\perp}$, the Boer-Mulders function $h_{1}^{\perp}$, the pretzelosity function $h_{1 T}^{\perp}$ and the so-called worm-gear functions $h_{1 L}^{\perp}$ and $g_{1 T}^{\perp}$.

The HERMES experiment at HERA has played a pioneering role in these studies, by measuring observables (azimuthal asymmetries) sensitive to these (and also some higher-twist) TMDs, in some cases providing the first evidence of non-zero effects. Most of the HERMES results are confirmed by other experiments (COMPASS, CLAS, etc), though intriguing differences were observed in some cases. High-precision results from the next generation high-luminosity and large-acceptance experiments (at present or upgraded facilities and, possibly, at the future Electron-Ion Collider) will greatly improve our understanding of the nucleon structure in terms of TMDs.

Among the TMDs, particularly interesting is the Sivers function. It describes the correlation between the quark transverse momentum and the transverse spin of the nucleon. The interest in this TMD, proposed more than 20 years ago [4] to explain the large single-spin asymmetries observed in pion production in the collision of unpolarized with transversely polarized nucleons, suddenly increased after it was demonstrated [5] to be linked to the quark and gluon orbital angular momentum, the main still unmeasured contribution to the nucleon spin. It is naive-T-odd, i.e. it requires final-state interactions (FSIs) of the ejected quark with the color field of the nucleon remnant. These FSIs are formally introduced in the definition itself of the Sivers function through a gauge link. In general, gauge links are processdependent and this leads to the remarkable fact that naive-T-odd TMDs (such as the Sivers and the Boer-Mulders functions) are not universal. In particular, they are expected to have opposite sign when measured in DrellYan processes. The experimental verification of this direct QCD prediction is eagerly awaited. The first evidence for a non-zero Sivers function was reported by the HERMES Collaboration in 2005 [2], using an unpolarized $27.5 \mathrm{GeV}$ electron beam and a transversely polarized hydrogen target. These amplitudes are sensitive to the convolution of the Sivers function $f_{1 T}^{\perp}$ and the unpolarized fragmentation function $D_{1}$ :

$$
A_{U T}^{\sin \left(\phi-\phi_{S}\right)} \propto \sum_{q} e_{q}^{2} f_{1 T}^{\perp, q}\left(x, p_{T}^{2}\right) \otimes D_{1}^{q}\left(z, k_{T}^{2}\right) .
$$

Here, $\phi$ and $\phi_{S}$ are, respectively, the azimuthal angle of the detected hadron and of the target transverse polarization with respect to the lepton scattering plane and about the virtual-photon direction, $z$ denotes the fraction of the energy of the exchanged virtual photon carried by the produced hadron (in 
this case a pion), and $p_{T}$ and $k_{T}$ represent the transverse momentum of the struck quark and that of the fragmenting quark, respectively.

In particular, HERMES observed large positive amplitudes for $\pi^{+}$ and amplitudes consistent with zero for $\pi^{-}$. The results were provided in 1-dimensional (1D) projections as a function of $x, z$ or $P_{h \perp}$. The vanishing amplitudes for $\pi^{-}$require cancellation effects, e.g., from a $d$-quark Sivers function opposite in sign to the $u$-quark Sivers function. The final HERMES results [6], based on the full statistics available, confirm the main features of the early results for charged pions and include results for neutral pions and charged kaons (Fig. 1). The amplitudes for $K^{+}$are large and positive while those for $\pi^{0}$ and $K^{-}$are slightly positive. Unexpectedly, the $K^{+}$ amplitudes are larger than the $\pi^{+}$ones. This observation can be ascribed, for instance, to a significant role of the sea quarks or to possible higher-twist contributions in kaon production. Recently, the data analysis was refined and the relevant asymmetry amplitudes were extracted in a 3-dimensional (3D) binning in $x, z$ and $P_{h \perp}$. As an example, Fig. 2 shows the Sivers amplitude for $\pi^{+}$as a function of $P_{h \perp}$ in simultaneous bins in $x$ and $z$. The 3D binning allows to map the kinematic dependence of the asymmetry amplitudes in a greatly detailed way. For instance, the rise of the Sivers amplitude for $\pi^{+}$as a function of $P_{h \perp}$, as observed in the conventional 1D projection (Fig. 1), reveals non-trivial kinematic dependencies in $x$ and $z$ when observed in the $3 \mathrm{D}$ representation (Fig. 2), where most of the rise is clearly localized in the high- $x$ and high- $z$ regions. Recently, the HERMES experiment has also measured single and double-spin asymmetries in SIDIS for protons and antiprotons. Figure 3 shows the Sivers amplitudes for protons and antiprotons in 1D bins in $x, z$ and $P_{h \perp}$. While the antiproton amplitude is consistent with zero within the experimental uncertainties, the proton data exhibit a small but significantly positive amplitude. This constitutes, at present, the first measurement of a non-zero Sivers effect for protons in SIDIS.

The experimental access to GPDs is so far obtained from the measurements of hard exclusive lepton-nucleon scattering processes. These processes are described by four leading-twist, quark chirality conserving GPDs $(H, E$, $\widetilde{H}$, and $\widetilde{E}$ ). The cleanest process to probe GPDs is Deeply Virtual Compton Scattering (DVCS), $\ell+N \rightarrow \ell+N^{\prime}+\gamma$, where a real photon is emitted by the struck quark in the nucleon. During the last decade the DVCS process was extensively studied both theoretically and experimentally. Currently there exist several measurements by HERMES, H1, ZEUS, CLAS and Hall-A experiments. The Bethe-Heitler (BH) process, where a real photon is radiated 

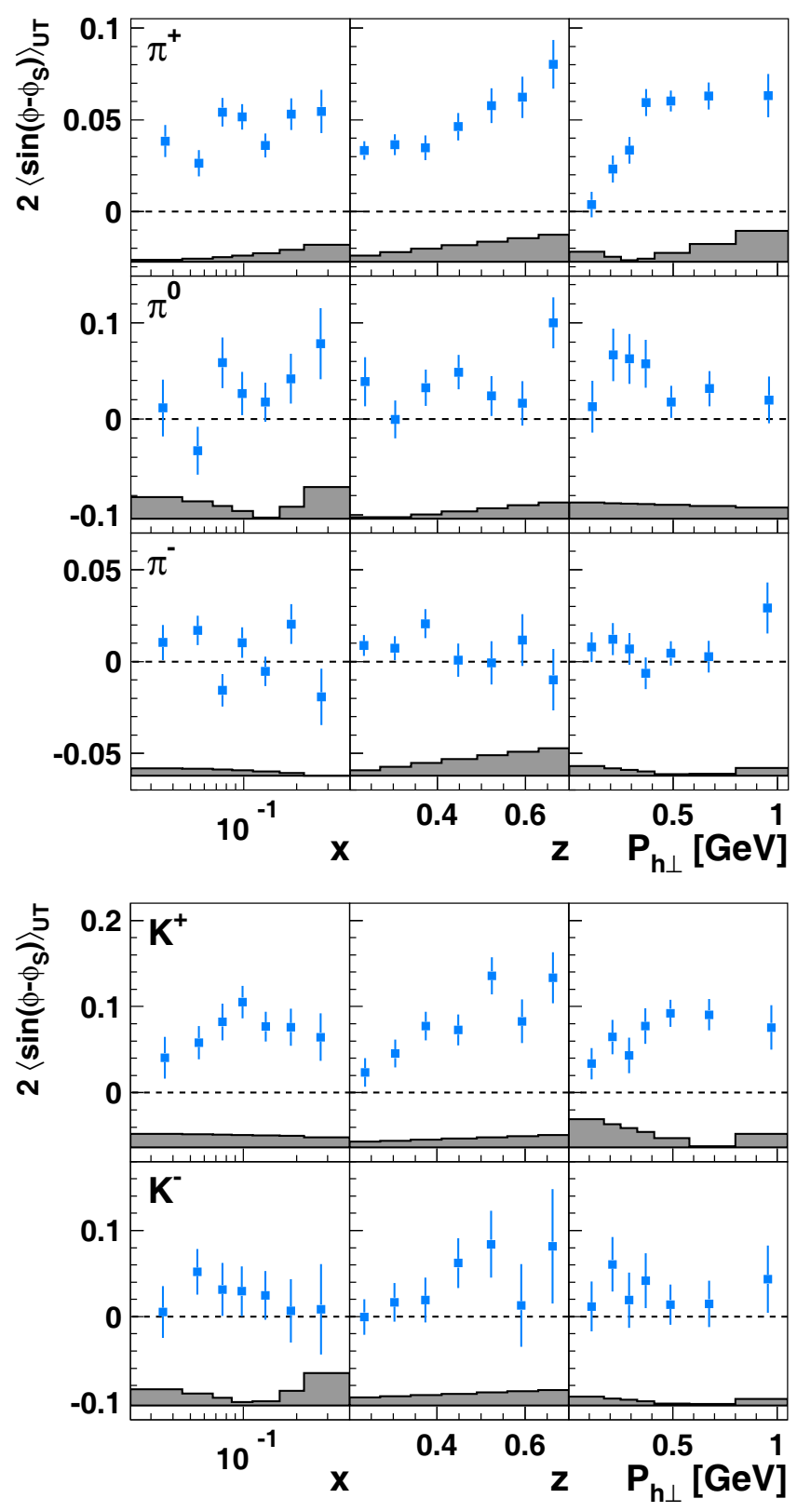

Figure 1: Sivers amplitudes for pions (top) and charged kaons (bottom) as a function of $x, z$, or $P_{h \perp}$. A $7.3 \%$ scale uncertainty, not shown, arises from the accuracy in the measurement of the (transverse) target polarization. 


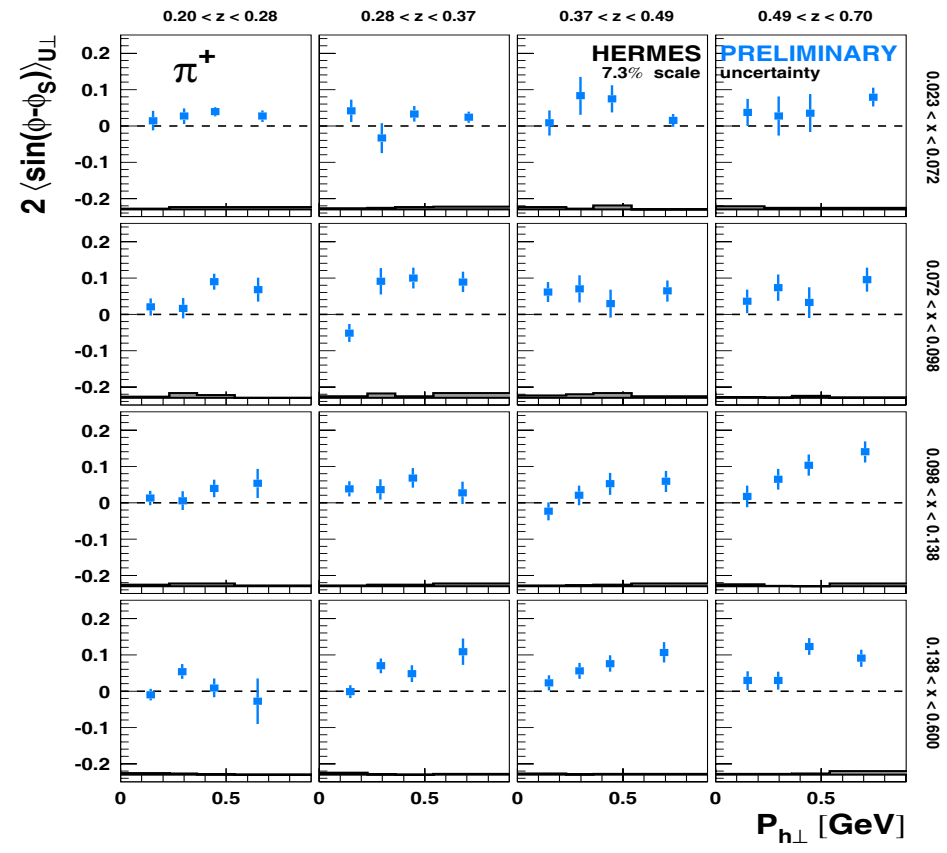

Figure 2: Sivers amplitudes for $\pi^{+}$in 3-dimensional binning in $x, z$ and $P_{h \perp}$.

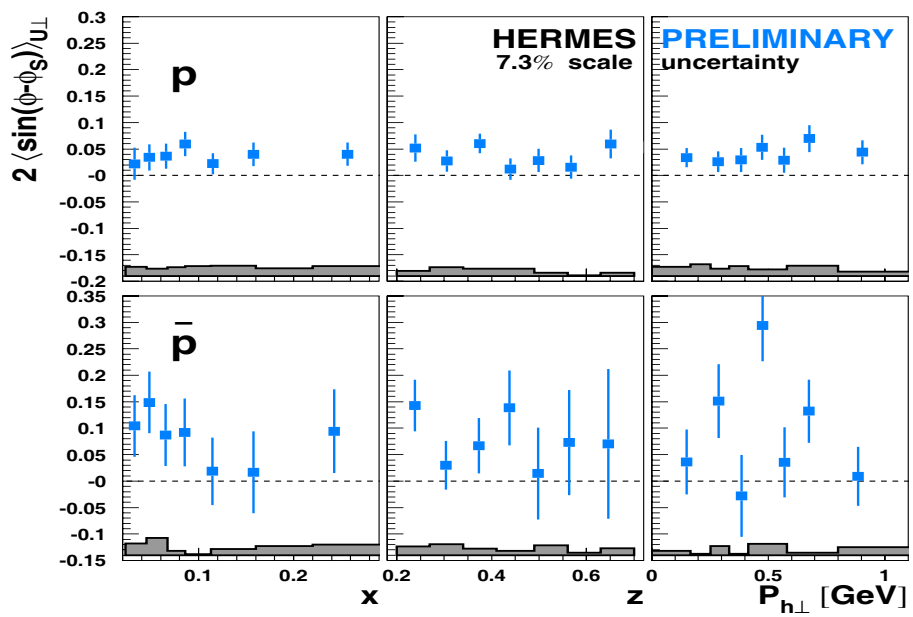

Figure 3: Sivers amplitudes for protons/anti-protons as a function of $x, z$ and $P_{h \perp}$. 


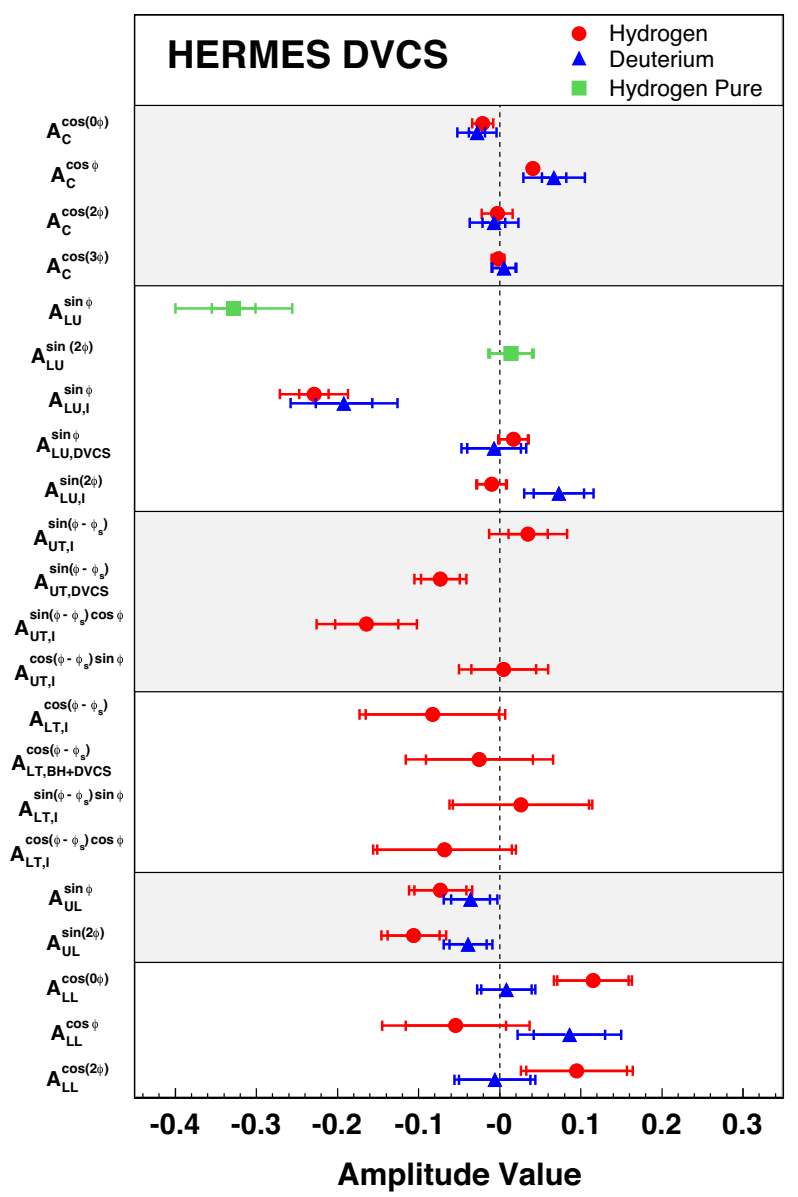

Figure 4: Overview of DVCS amplitudes over the entire HERMES kinematic range.

by the incoming or outgoing lepton, has the same initial and final state as the DVCS process. These two processes are thus experimentally indistinguishable, and therefore the total cross-section of hard lepto-production of real photons contains an interference term $\mathcal{I}$, which depends on the charge of the lepton beam: $\sigma \propto\left|\mathcal{T}_{B H}\right|^{2}+\left|\mathcal{T}_{D V C S}\right|^{2}+\mathcal{I}$. The individual terms of the cross-section can be decomposed into Fourier harmonics in the relevant azimuthal angles. Different Fourier components depend on different Compton Form Factors (CFFs), which in turn are convolutions of hard scattering amplitudes with the corresponding GPDs.

At HERMES, the DVCS process is accessed through measurements of cross-section asymmetries that appear in the azimuthal distributions of final 
state photons. Using data collected with longitudinally polarized electron and positron beams and unpolarized, longitudinally and transversely polarized hydrogen and deuterium targets, it was possible to measure asymmetries with respect to beam charge, beam polarization and target polarization alone and also with respect to their different combinations. An overview of all extracted azimuthal asymmetry amplitudes corresponding to the entire HERMES kinematics is presented in Fig. 4 for both hydrogen and deuterium targets. The amplitudes of the beam-helicity and beam-charge asymmetries $A_{L U}^{D V C S}(\phi), A_{L U}^{I}(\phi)$ and $A_{C}(\phi)$ are presented in the top panels. Significant non-zero $\cos (\phi)$ and $\sin (\phi)$ amplitudes are observed respectively for the beam-charge $A_{C}(\phi)$ and beam-helicity $A_{L U}^{I}(\phi)$ asymmetries. The results for the $\sin (\phi)$ amplitude of the charge-averaged beam-helicity asymmetry $A_{L U}^{D V C S}(\phi)$ are consistent with zero. In the bottom panels, the results of the longitudinal single-target-spin $A_{U L}(\phi)$ and double-spin $A_{L L}(\phi)$ asymmetries are presented. Also shown are the leading amplitudes of transverse singletarget-spin and double-spin asymmetries, measured on Hydrogen target. The amplitudes $A_{U T, I}^{\sin \left(\phi-\phi_{S}\right) \cos (\phi)}$ and $A_{L T, I}^{\sin \left(\phi-\phi_{S}\right) \sin (\phi)}$ are significantly negative and consistent with zero, respectively. Full references for the HERMES DVCS results can be found in [7-9].

\section{References}

[1] K. A. Olive et al. (PDG), Chin. Phys. C 38, 090001 (2014).

[2] A. Airapetian et al. (HERMES), Phys. Rev. Lett. 94, 012002 (2005).

[3] A. Bacchetta et al., JHEP 02, 093 (2007).

[4] D. W. Sivers, Phys. Rev. D 41, 83 (1990).

[5] S. J. Brodsky et al. Phys. Lett. B 530, 99 (2002).

[6] A. Airapetian et al. (HERMES), Phys. Rev. Lett. 103, 152002 (2009).

[7] A. Airapetian et al. (HERMES), JHEP 07, 032 (2012), JHEP 06, 019 (2010), JHEP 06, 066 (2008), Phys. Lett. B 704, 15 (2011).

[8] A. Airapetian et al. (HERMES) Nucl. Phys. B 829, 1 (2010), Nucl. Phys. B 842, 265 (2011).

[9] A. Airapetian et al. (HERMES), JHEP 10, 042 (2012). 